Pacific Journal of Mathematics

NONOPENNESS OF THE SET OF THOM-BOARDMAN MAPS 


\title{
NONOPENNESS OF THE SET OF THOM-BOARDMAN MAPS
}

\author{
LESLIE C. WILson
}

1. Introduction. In this paper, we show that the set of all $C^{\infty}$ Thom-Boardman maps from an $n$-dimensional manifold is not open iff corank two singularities occur generically. The latter is known to occur iff either $n \leqq p$ and $2 p \leqq 3 n-4$ or $n>p$ and $2 p \geqq n+4$. In the course of the proof, we establish a variation of Mather's Multitransversality Theorem: we show that jets have extensions which are multitransverse to given submanifolds of the jet bundle except possibly at the original jet. As an application of this extension theorem, we show that, in Mather's "nice range of dimensions," each jet $z$ has a representative $f\left(z=j^{k} f(x)\right)$ such that $f$ is infinitesimally stable on a deleted neighborhood of $x$.

First we recall some properties of Thom-Boardman singularities; for more details, the reader is referred to [1], [11] and [3]. In this paper $N$ (respectively $P$ ) will always be an $n$ (respectively $p$ ) dimensional manifold without boundary. There is a finite partition of the jet bundle $J^{k}(N, P)$ into embedded submanifolds $S^{I}$, called ThomBoardman Singularities, each $I$ a nonincreasing sequence of nonnegative integers. Consider $f$ in $C(N, P)$, the set of smooth maps from $N$ to $P$. Let $S^{I}(f)$ denote $\left(j^{k} f\right)^{-1} S^{I}, j^{k} f$ the jet extension of $f$. Then $j^{k} f \pitchfork S^{i}$ implies that $S^{i}(f)$ is the set of points at which $\operatorname{dim} \operatorname{ker} T f=$ $i ; j^{k} f \pitchfork S^{i, j}$ implies $j^{k} f \pitchfork S^{i}$ and $S^{i, j}(f)=S^{j}\left(f \mid S^{i}(f)\right)$, etc. We call $f$ a Thom-Boardman map if $j^{k} f \pitchfork S^{I}$ for all $I$, for all $k$. By Thom's Transversality Theorem, the set of Thom-Boardman maps is residual (i.e., is a countable intersection of open, dense sets), hence is dense, in the Whitney $C^{\infty}$ topology.

THEOREM 1.1. The Thom-Boardman maps form an open subset of $C(N, P)$ iff either $n \leqq p$ and $2 p>3 n-4$ or $n>p$ and $2 p<n+4$.

Let $r=\max (n-p, 0)$, let $S_{i}$ denote $S^{i+r}$ (jets of corank $i$ ), let $S_{i, j}$ denote $S^{i+r, j}$, etc. The condition on $n$ and $p$ in the theorem is precisely the condition that the codimension (abbreviated cod) of $S_{2}$ be greater than $n$, hence that maps cannot take on $S_{2}$ singularities transversally.

If $\operatorname{cod} S_{2}$ is greater than $n$, then a map is Thom-Boardman iff it is transverse to all Morin singularities (which are the $S_{1 ; k}$, where $1 ; k$ means $1, \cdots, 1,0,1$ occurring $k$ times). In this case, a map is 
Thom-Boardman iff its germ at each point is stable. It follows that the set of Thom-Boardman maps is open in these dimensions. It is helpful in understanding the proof of our theorem to consider a more general method for demonstrating the openness of a set of maps defined by transversality conditions.

A stratification of a closed set $X$ in a manifold $M$ is a locally finite partition of $X$ into embedded submanifolds, called strata, satisfying the "condition of the frontier": if $S$ and $T$ are strata and $S \cap \mathrm{Cl}(T) \neq \varnothing$ (Cl denotes closure), then $S \subset \mathrm{Cl}(T)$. Whitney's condition $A$ requires that, for $x \in S$ and $x_{n} \in T$, if $x_{n}$ converges to $x$ and $T_{x_{n}}$ (= tangent space to $T$ at $x_{n}$ ) converges to some $L$ in the Grassman bundle, then $S_{x} \subset L$.

The following proposition is folklore:

Proposition 1.2. Suppose $X$ is a closed subset of $J^{k}(N, P)$ and $\mathscr{S}$ is a stratification of $X$ satisfying Whitney's condition $A$. Then $\left\{f: j^{k} f \pitchfork S\right.$ for all $\left.S \in \mathscr{S}\right\}$ is open in $C(N, P)$ with the Whitney $C^{\infty}$ topology.

Using the method for constructing Whitney stratifications given in [13] together with the fact that the Morin singularities $S_{1 ; k}$ are orbits of a smooth group action on $J^{k}(N, P)$ (the group being $\mathscr{K}$; see [7] and [8]), one can show that there is a stratification of $J^{k}(N, P)$ satisfying condition $A$ with the Morin singularities among the strata. In the case $\operatorname{cod} S_{2}>n$, the Thom-Boardman maps are precisely those which are transversal to all those strata, hence form an open set by Proposition 1.2.

However, we will give examples in $\S 2$ showing that $S_{2,0} \cap$ $\mathrm{Cl}\left(S_{1 ; k}\right) \neq \varnothing$ for all $k$. Since $\operatorname{cod} S_{1 ; k}>\operatorname{cod} S_{2}$ if $k$ is sufficiently large, condition $A$ cannot hold between $S_{1 ; l c}$ and $S_{2}$. (In fact, it is shown in [5] that condition $A$ already fails between $S_{1,1}$ and $S_{2,0}$ ) Our examples will also show that the condition of the frontier fails.

Let ${ }_{t} S_{I}$ denote those $z$ in $S_{I}$ such that, for all $f$ with $j^{k} f(x)=z$, $j^{k} f \pitchfork S_{I}$ at $z$. These jets are called the transversal elements of $S_{I}$. Proposition 2.1 implies that ${ }_{t} S_{2,0} \cap \mathrm{Cl}\left(S_{1 ; k}\right) \neq \varnothing$ for all $k$ if $\operatorname{cod} S_{2} \leqq n$. Consider a $z$ in this intersection. By the Transversal Extension Theorem (3.1) there is a Thom-Boardman map $f$ such that $j^{k} f(x)=z$ for some $x$. We pick $k$ large enough that $\operatorname{cod} S_{1 ; k}>n$. There are $z_{i} \in S_{1 ; k}$ with $z_{i}$ converging to $z$ in $J^{k}(N, P)$. Lemma 3.5 demonstrates that there are $f_{i} \in C(N, P)$ such that $j^{k} f_{i}\left(x_{i}\right)=z_{i}$ and $f_{i} \rightarrow f$ in the Whitney $C^{\infty}$ topology; these $f_{i}$ can not be Thom-Boardman, hence Theorem 1.1 will be proven.

2. Contact classes in $S_{2,0}$. First we describe Mather's algorithm 
for determining in which $S^{t}$ a jet $z$ lies (see [11]). Let $A_{m}$ be the formal power series algebra in $n$ variables over $\boldsymbol{R}$. Let $m_{n}$ denote the unique maximal ideal of $A_{n}$ (i.e., all formal power series with zero constant term). If $f$ is a map germ at 0 from $\boldsymbol{R}^{n}$ to $\boldsymbol{R}^{p}$, let $I(f)$ denote the ideal in $A_{n}$ generated by the Taylor series at 0 of the component functions of $f$. The rank of an ideal $I$ is by definition the dimension of $\left(m_{n}^{2}+I\right) / m_{n}^{2}$ as a real vector space, and crk $I=$ $n-\operatorname{rank} I$. Suppose $\operatorname{rank} I=r$; pick $f$ such that $I=I(f)$. Let $\delta I$ be the ideal generated by $I$ and the $r+1$ by $r+1$ subdeterminants of the Jacobian matrix of $f ; \delta I$ depends only on $I$, not on $f$. The Boardman symbol of $I$, or of $f$ if $I=I(f)$, is $i_{1}, i_{2}, \cdots$ where $i_{1}=$ $\operatorname{crk} I, i_{2}=\operatorname{crk} \delta I, i_{3}=\operatorname{crk} \delta(\delta I)$, etc. Mather shows that, if $z=j^{k} f(x)$, then $z$ is in $S^{I}$, where $I$ consists of the first $k$ terms of the Boardman symbol of any coordinate representation of $f$ near $x$.

The algebra of a $k$-jet $z$ is $A_{n} /\left(I(g)+m_{n}^{k+1}\right)$ where $g$ is a coordinate representation of a map whose $k$-jet is $z$; this algebra is only determined up to algebra isomorphism. If $f$ is a germ at $x$, let $V_{k}(f)$ denote the set of $k$-jets whose algebras are isomorphic to that of $j^{k} f(x) ; V_{l}(f)$ is called the contact class of $j^{k} f(x)$, and is the orbit of the action of the group $\mathscr{\%}$ (see [8]). From the previous paragraph we see that each $S_{I}$ is a union of contact classes.

Next we show that $S_{2,0} \cap \mathrm{Cl}\left(S_{1 ; k}\right) \neq \varnothing$ for all $k$. First suppose $n \leqq p$. It is easy to show using Mather's algorithm for computing the Boardman sequence that the Morin singularity $S_{1, k}$ in $J^{l}, l>l$, is equal to $V_{l}\left(x^{k+1}\right)$ (compare with $\S 3$ of Chapter VII of [3]). Also one sees that $S_{2,0} \subset J^{l}$ contains $V_{l}(x y), l>1$. Let

$$
F_{u}: R \times R \times \boldsymbol{R}^{n-2} \longrightarrow \boldsymbol{R} \times \boldsymbol{R} \times \boldsymbol{R}^{p-n} \times \boldsymbol{R}^{n-2}
$$

be defined by

$$
F_{u}(x, y, z)=\left(x y+u x^{k+1}, u y, 0, z\right) ;
$$

$j^{l} F_{u}(0) \in V_{l}\left(x^{k \cdot 1}\right)$ if $u \neq 0$ and $j^{l} F_{0}(0) \in V_{l}(x y)$ for $l>k$. Thus $V_{l}(x y) \subset$ $\mathrm{Cl}\left(S_{1 ; k k}\right)$ for all $k<l$.

Now suppose $n>p$. Let

$$
F_{u}: R \times R \times \boldsymbol{R}^{n-\rho} \times \boldsymbol{R}^{p-2} \longrightarrow \boldsymbol{R} \times \boldsymbol{R} \times \boldsymbol{R}^{p-2}
$$

be defined by

$$
F_{u}(x, y, w, z)=\left(x y+u x^{k+1}+\sum_{i=1}^{n-1 p} w_{i}^{2}, u y, z\right) ;
$$

then $j^{l} F_{u}(0) \in V_{l}\left(x^{k+1}+\sum_{i=1}^{n-p} w_{i}^{q}\right) \subset S_{1 ; k}$ if $u \neq 0$ and

$$
j^{l} F_{0}(0) \in V_{l}\left(x y \div \sum_{i=1}^{n-p} w_{i}^{2}\right) \subset S_{2,0} \text { for } l>l i
$$


Thus

$$
V_{l}\left(x y+\sum_{i=1}^{n-p} w_{i}^{2}\right) \subset \operatorname{Cl}\left(S_{1 ; k}\right) \text { for all } k<l .
$$

We wish to show that ${ }_{t} S_{2,0} \cap \mathrm{Cl}\left(S_{1 ; k}\right) \neq \varnothing$, for all $k$, if $\operatorname{cod} S_{2,0} \leqq n$. In fact, this follows by applying the next proposition to the contact class $V_{l}\left(x y+\sum_{i=1}^{n-p} w_{i}^{2}\right)$.

Proposition 2.1. Suppose cod $S_{2,0}$ is no greater than n. Then each contact class in $S_{2,0} \subset J^{l}$ contains an element of ${ }_{t} S_{2,0}$.

Proof. Suppose $j^{l} f(x)=z \in S_{2}=S^{r+2}$. The intrinsic second derivative of $f$ at $x$ (see $\S 3$ and $\S 4$ of Chapter VI of [3] and $\S 2$ of [10]) is a bilinear map $D^{2} f_{x}: N_{x} \times K \rightarrow C$, where $K=\operatorname{ker} D f_{x}$ and $C=\operatorname{cok} D f_{x}$. Local coordinates can be chosen centered about $x$ and $f(x)$ so that $f$ has the form $f\left(x_{1}, \cdots, x_{n}\right)=\left(x_{1}, \cdots, x_{s}, g\right)$, where $s=n-r-2$ is the rank of $f$, and

$$
g\left(x_{1}, \cdots, x_{n}\right)=\sum_{i, j=1}^{s} a_{i j} x_{i} x_{j}+\sum_{j=1}^{s} \sum_{k=s+1}^{n} b_{k j} x_{k} x_{j}+\sum_{k, l=s+1}^{n} c_{k l} x_{k} x_{l}
$$

where the coefficients are in $C\left(\boldsymbol{R}^{n}, \boldsymbol{R}^{p-S}\right)$. If we evaluate the coefficients at 0 , then (2.2) reduces to a vector-valued quadratic form. Viewed in these coordinates, $D^{2} f_{x}$ is the last two terms of this quadratic form; $d^{2} f_{x}=D^{2} f_{x} \mid K \times K$ is the last term and $D^{2} f_{x} \mid K^{\perp} \times K$ is the second term ( $K^{\perp}$ is the set $\left.x_{s+1}=\cdots=x_{n}=0\right)$.

$D^{2} f_{x}$ can also be viewed as a map from $N_{x}$ to $\operatorname{Hom}(K, C)$, and $\operatorname{Hom}(K, C)$ is canonically isomorphic to the normal space to $S_{2}$. Thus $z$ is in ${ }_{t} S_{2}$ iff $D^{2} f_{x}: N_{x} \rightarrow \operatorname{Hom}(K, C)$ is surjective. Furthermore, $z$ is in $S_{2,0}$ iff $d^{2} f_{x}: K \rightarrow \operatorname{Hom}(K, C)$ is injective.

Assume $z$ is in $S_{2,0}$. Then the image of $d^{2} f_{x}: K \rightarrow \operatorname{Hom}(K, C)$ has dimension $n-s$. Since

$$
\operatorname{dim} \operatorname{Hom}(K, C)=\operatorname{cod} S_{2} \leqq n, \operatorname{dim}\left(\operatorname{Hom}(K, C) / d^{2} f_{x}(K)\right)
$$

is less than or equal to $s=\operatorname{dim} K^{\lrcorner}$. Since we can choose the coefficient functions in the second term of (2.2) arbitrarily without affecting the ideal, hence the contact class, of $f$, we can choose an $f^{\prime}$ contact equivalent to $f$ so that $d^{2} f_{x}^{\prime}=d^{2} f_{x}$ and $D^{2} f_{x}^{\prime} \mid K^{\perp}$ spans $\operatorname{Hom}(K, C) \bmod d^{2} f_{x}^{\prime}(K)$. Hence $D^{2} f_{x}^{\prime}$ is surjective; hence $j^{l} f^{\prime}(x)$ is in ${ }_{t} S_{2}$.

While it is not relevant to this paper, we would like to mention that Mather has proved the following (Theorem 6.1 of [9]): if $U$ is a $C^{2}$ submanifold of $J^{k}(N, P)$ and $\operatorname{cod} U \leqq n$, then the set of transversal points (i.e., ${ }_{t} U$ ) is dense in $U$. 
3. Transversal extensions of jets. In this section we establish a variation of the Multijet Transversality Theorem (due to Mather [9]; see also [3]) and give some applications. Then we complete the proof of Theorem 1.1.

Suppose $X \subset N$ and $h: X \rightarrow J^{k}(N, P)$ is a section. Let $A=$ $\left\{f \in C(N, P): j^{k} f \mid X=h\right\} ; A$ is a closed subset of $C(N, P)$ in the $C^{k}$, hence in the $C^{\infty}$, topology. Furthermore, $A$ is a Baire space (see Theorem $4.4 \mathrm{~b}$ of Chapter 2 of [4]), i.e., every residual set (= countable intersection of open, dense sets) is dense.

Now suppose $X$ is closed in $N$. Let $\pi:{ }_{s} J^{k}(N, P) \rightarrow N^{(s)} \times P^{s}$ denote the $s$-fold $k$-jet bundle and let $X^{(s)}$ be the set of $s$-tuples with at least one component in $X ; X^{(s)}$ is closed in $N^{(s)}$. Suppose $W$ is an immersed submanifold of $\pi_{1}^{-1}\left(N^{(s)}-X^{(s)}\right)$, where $\pi_{1}$ is the composition of $\pi$ with the projection of $N^{(s)} \times P^{s}$ on its first factor. Let $A^{t}=$ $\left\{f \in A:{ }_{s} j^{k} f \mid\left(N^{(s)}-X^{(s)}\right) \pitchfork W\right\}$.

Proposition 3.1. (Multijet Transversal Extension Theorem.) $A^{t}$ is residual in $A$.

Proof. $W$ can be covered by a countable collection of compact, codimension 0 submanifolds $M_{i}$ (with boundary); furthermore, the $M_{i}$ may be chosen so that, for each $i$, there exist relatively compact, mutually disjoint coordinate patches $U_{i, 1}, \cdots, U_{i, s}$ whose closures are contained in $N-X$ and $V_{i, 1}, \cdots, V_{i, s}$ in $P$ such that $\pi\left(M_{i}\right) \subset U_{i, 1} \times$ $\cdots \times U_{i, s} \times V_{i, 1} \times \cdots \times V_{i, s}$.

Let $B_{i}=\left\{f \in C(N, P):{ }_{s} j^{k} \pitchfork W\right.$ on $\left.M_{i}\right\}$ and let $A_{i}=B_{\imath} \cap A ; A_{i}$ is open in $A$ since $B_{i}$ is open (see II. 4.14 of [3]). Pick any neighborhood $U$ of $f$. Exactly as in the proof of the Multijet Transversality Theorem ([9] or [3]), there is a $g$ in $B_{i} \cap U$ which agrees with $f$ outside $U_{i, 1} \cup \cdots \cup U_{i, s}$; thus $g$ is in $A_{i} \cap U$. Hence $A_{i}$ is dense in $A ; A^{t}=\cap A_{i}$ is residual.

CoROLlary 3.2. If $\left\{W_{i}\right\}$ is a countable collection of immersed submanifolds of $\pi_{1}^{-1}\left(N^{(s)}-X^{(s)}\right)$, then $\left\{f \in A: j^{k} f \mid\left(N^{(s)}-X^{(s)}\right) \pitchfork W_{i}\right.$ for all i\} is residual.

In Mather's nice range of dimensions ([9] and [10]), ${ }_{s} J^{k}(N, P)$ is the disjoint union of finitely many contact classes $C_{i}$ and finitely many other manifolds $M_{i}$ of codimension greater than $n$. Thus, in these dimensions, a map is multitransverse to all contact classes iff it is multitransverse to the $C_{i}$ 's and $M_{i}$ 's.

Mather shows in [9] that if $f$ is multitransverse to all contact classes and if $f \mid C(f)(C(f)$ is the set of points in $N$ at which the rank of $f$ is less than $p$ ) is proper, then $f$ is infinitesimally stable; 
if in addition $f$ is proper, then $f$ is stable.

COROLlaRy 3.3. In the nice range of dimensions, for every $k$ jet $z$ there is a map $f$ defined on a neighborhood $U$ of $x$ in $N$ such that $j^{k} f(x)=z$ and $f \mid(U-\{x\})$ is infinitesimally stable; if $n \leqq p$, then $f \mid(U-\{x\})$ is stable.

Proof. First, we can find an $f$ on a $U$ so that $j^{k} f(x)=z$ and $f$ is finite (see Example 5.2 of Chapter VIII of [12] and $\S 2$ of Chapter VII of [3]). Since we are in the nice range of dimensions and finite maps form an open set, we can assume that $f$ is multitransverse to all contact classes on $U-\{x\}$. Since $f$ is finite, shrinking $U$ if necessary, $f \mid(U-\{x\})$ will be proper on $C(f)$; if $n \leqq p, f$ will be proper on all of $U-\{x\}$.

Note that, by Lemma 4.4 of [9] and Theorem 1.13 of [7], if $j^{p} f$ is transverse to the contact class of $j^{p} f(x)$, then the germ of $f$ at $x$ is stable.

A germ $f_{x}$ is finitely-determined if, for some $l$, every germ $g_{x}$ having the same $l$-jet as $f_{x}$ at $x$ can be transformed into $f_{x}$ by local coordinate changes. If $f_{x}$ is finitely-determined, then for every representative $f$ of $f_{x}$ there is a neighborhood $U$ of $x$ such that $f \mid(U-\{x\})$ is multitransverse to all contact classes (see [2]); the converse is true if $f$ is complex analytic. This suggests the conjecture that, in the nice range of dimensions, every jet has a finitelydetermined representative. C. T. C. Wall has recently confirmed that this is true in a range of dimensions slightly larger than the nice range.

We return to the proof of Theorem 1.1.

Corollary 3.4. If cod $S_{2} \leqq n$, then for each $x \in N$ and all $k \geqq 1$ there is a Thom-Boardman map $f$ such that $j^{k+1} f(x) \in S_{2,0} \cap \operatorname{Cl}\left(S_{1: k}\right)$.

Proof. We know that $V_{k+1}\left(x y+\sum_{i=1}^{n-p} w_{i}^{2}\right)$ is contained in $S_{2,0} \cap$ $\mathrm{Cl}\left(S_{1 ; k}\right)$. Choose in this contact class a transversal element $z$ of $S_{2}$ so that $\pi_{1}(z)=x$. Let $A=\left\{f \in C(N, P): j^{k+1} f(x)=z\right\}$. Applying Corollary 3.2 to the case $s=1$ and $\left\{W_{i}\right\}$ the Thom-Boardman singularities, we see that there is an $f$ such that $j^{k+1} f(x)=z$ and away from $x j^{k+1} f$ is transverse to all Thom-Boardman singularities. Thus $f$ is a Thom-Boardman map.

LemmA 3.5. Suppose $z_{m} \rightarrow j^{k} f(x) \in J^{k}(N, P)$. Then there are maps $f_{m}$ and points $x_{m}$ such that $j^{k} f_{m}\left(x_{m}\right)=z_{m}$ and $f_{m} \rightarrow f$ in the Whitney $C^{\infty}$ topology. 
Proof. Necessarily $\pi\left(z_{m}\right)=\left(x_{m}, y_{m}\right)$ converges to $\pi(z)=(x, y)$. Choose coordinate charts $(U, r)$ about $x$ and $(V, l)$ about $y$ such that $r(U)=\boldsymbol{R}^{n}, r(x)=0, r\left(x_{m}\right)=u_{m}$ and $l(V)=\boldsymbol{R}^{p}$. Choose any maps $\boldsymbol{g}_{m}$ such that $j^{k} g_{m}\left(x_{m}\right)=z_{m}$. Let $g_{m}^{\prime}=l \circ g_{m} \circ \gamma^{-1}$ and $f^{\prime}=l \circ f \circ \gamma^{-1}$. Let $p_{m}$ be a polynomial of degree $k_{k}$ expanded about $u_{m}$ such that $j^{k} p_{m}\left(u_{m}\right)=$ $j^{k}\left(g_{m}^{\prime}-f^{\prime}\right)\left(u_{m}\right)$. Since $j^{k} p_{m}\left(u_{m}\right)$ converges to the zero jet at 0 , the coefficients of the $p_{m}$ 's go to 0 . For any compact set $K \subset \boldsymbol{R}^{n}$, there is a $C$ such that $\left|u-u_{m}\right|^{i}<C$ for all $u \in K, m>0$ and $0<i \leqq k$. Thus $p_{m} \rightarrow 0$ uniformly on $K$, as does each of its derivatives. Pick $a \in C\left(\boldsymbol{R}^{n}, \boldsymbol{R}\right)$ such that $a=1$ on a neighborhood of 0 and $a=0$ off a compact set. Let $f_{m} \mid U=l^{-1}\left(a p_{m}+f^{\prime}\right) \circ r$ and $f_{m} \mid(N-U)=f$. Then $f_{m}, f$ in the Whitney $C^{c o}$ topology and $j^{k} f_{m}\left(x_{m}\right)=z_{m}$.

Choose $k$ sufficiently large that $\operatorname{cod} S_{1: k}>n$. Choose $f$ as in Corollary 3.4. By the preceding lemma, $f$ is the limit of maps $f_{m}$ having $S_{1 ; k}$ singularities. Necessarily $f_{m}$ is not a Thom-Boardman map. Thus the proof of Theorem 1.1 is complete.

EXAMPLE 3.6. Let $f(x, y, s, t)=(g(x, y)+s x+t y, x y, s, t)$, where $g$ is in $m_{2}^{2}$; then $j^{2} f(0)$ is in ${ }_{t} S_{2,0}$. If we take $g(x, y)=x^{3}+y^{3}$, then $f$ is a Thom-Boardman map and is the limit of maps which, having $S_{1: 5}$ singularities, are not Thom-Boardman. The computational details involved in this example are worked out in [6].

\section{REFERENCES}

1. J. M. Boardman. Singularities of differentiable maps, Publ. Math. I.H.E.S., 33 (1967). 21-57.

2. T. Gaffney, Properties of Finitely Determined Germs, Thesis, Brandeis Univ., 1975.

3. M. Golubitsky and V. Guillemin, Stable Mappings and Their Singularities, SpringerVerlag, New York, 1973.

4. M. Hirsch, Differential Topology, Springer-Verlag, New York, 1976.

5. L. Lander, Stratification by orbits, with an application to $J^{2}(2,2)$, J. London Math. Soc., (2), 8 (1974), 443-450.

6. - Thesis, Universität Regensburg, 1972.

7. J. Mather, Stability of $C^{\infty}$ mappings, III: finitely determined map germs, Publ. Math. I.H.E.S., 35 (1968), 127-156.

8. - Stability of $C^{\infty}$ mappings, IV: classification of stable germs by $R$-algebras, Publ. Math. I.H.E.S., 37 (1969), 223-248.

9. - Stability of $C^{\infty}$ mappings, $V$ : transversality, Advances in Math., 4 (1970). 301-336.

10. Stability of $C^{\infty}$ mappings, VI: the nice dimensions, Proceedings of the Liverpool Singularities Symposium I, Lecture Notes in Math. No. 192, Springer-Verlag, New York, 1971.

11. - On Thom-Boardman Singularities, Dynamical Systems, Academic Press, New York, 1973.

12. J. C. Tougeron, Idéaux de Fonctions Différentiables, Ergebnisse Band 71, SpringerVerlag. New York. 1972. 
13. C. T. C. Wall, Regular Stratifications, Dynamial Systems-Warwick 1974, Lecture Notes in Math. No. 468, Springer-Verlag, New York, 1975.

Received July 18, 1978 and in revised form February 9, 1979.

UNIVERSITY OF HAWAII

HONOLULU, HI 96822 


\section{PACIFIC JOURNAL OF MATHEMATICS}

\section{EDITORS}

DONALD BABBITT (Managing Editor)

University of California

Los Angeles, California 90024

Hugo RossI

University of Utah

Salt Lake City, UT 84112

C. C. MOORE and ANDrew OGG

University of California

Berkeley, CA 94720
J. DUgundjI

Department of Mathematics University of Southern California Los Angeles, California 90007

R. FinN aNd J. Milgram Stanford University Stanford, California 94305

\section{ASSOCIATE EDITORS}

E. F. BECKENBACH

B. H. NeUMANN

F. WOLF

K. YosHIDA

\section{SUPPORTING INSTITUTIONS}

UNIVERSITY OF BRITISH COLUMBIA CALIFORNIA INSTITUTE OF TECHNOLOGY UNIVERSITY OF CALIFORNIA MONTANA STATE UNIVERSITY UNIVERSITY OF NEVADA, RENO NEW MEXICO STATE UNIVERSITY OREGON STATE UNIVERSITY UNIVERSITY OF OREGON
UNIVERSITY OF SOUTHERN CALIFORNIA STANFORD UNIVERSITY UNIVERSITY OF HAWAII UNIVERSITY OF TOKYO UNIVERSITY OF UTAH WASHINGTON STATE UNIVERSITY UNIVERSITY OF WASHINGTON 


\section{Pacific Journal of Mathematics}

\section{Vol. 84 , No. 1 \\ May, 1979}

Michael James Beeson, Goodman's theorem and beyond ...............

Robert S. Cahn and Michael E. Taylor, Asymptotic behavior of multiplicities

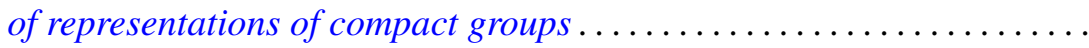

Douglas Michael Campbell and Vikramaditya Singh, Valence properties of

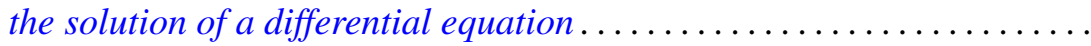

J.-F. Colombeau, Reinhold Meise and Bernard Perrot, A density result in spaces of Silva holomorphic mappings .....................

Marcel Erné, On the relativization of chain topologies .................

Le Baron O. Ferguson, Uniform and $L_{p}$ approximation for generalized

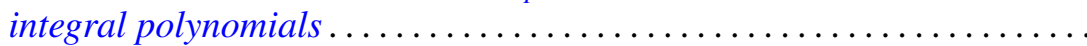

Kenneth R. Goodearl and David E. Handelman, Homogenization of regular rings of bounded index..................................

Friedrich Haslinger, A dual relationship between generalized

Abel-Gončarov bases and certain Pincherle bases .................

Miriam Hausman, Generalization of a theorem of Landau. .

Makoto Hayashi, 2-factorization in finite groups

Robert Marcus, Stochastic diffusion on an unbounded domain ........... 143

Isabel Dotti de Miatello, Extension of actions on Stiefel manifolds....

C. David (Carl) Minda, The hyperbolic metric and coverings of Riemann surfaces...

Somashekhar Amrith Naimpally and Mohan Lal Tikoo, On $T_{1}$-compactifications.

Chia-Ven Pao, Asymptotic stability and nonexistence of global solution for a semilinear parabolic equation ....

Shigeo Segawa, Harmonic majoration of quasibounded type ...

Sze-Kai Tsui and Steve Wright, The splitting of operator algebras ...

Bruce Williams, Hopf invariants, localization and embeddings of Poincaré complexes....

Leslie Wilson, Nonopenness of the set of Thom-Boardman maps ...

Alicia B. Winslow, There are $2^{\mathrm{c}}$ nonhomeomorphic continua in $\beta R^{n}-R^{n}$ 